\title{
ON THE CHOICE OF A CAPABILITY INDEX FOR ASYMMETRIC TOLERANCES
}

\author{
Daniel Grau \\ Laboratory of Applied Mathematics, CNRS UMR 5142 \\ IUT de Bayonne, Université de Pau et des Pays de l'Adour, \\ 3 Av. Jean Darrigrand, 64100 Bayonne, France \\ Email: daniel.grau@univ-pau.fr \\ Fax : $33(0) 559574379$
}

\begin{abstract}
For a process normally distributed and a target located at the mid-point of a twosided tolerance interval, Vännman suggests a family of indices depending on two parameters. In order to choose the parameters which allow to obtain an index that is sensitive to departures of the mean from the target, we need to take the properties of its estimator into account. For a target not located at the mid-point of the interval of tolerance, Chen and Pearn suggest a family of indices generalizing Vännman's. The moments of these indices are given, then their expected values and mean square errors are studied to choose an index that is sensitive to the deviations of the mean from the target.
\end{abstract}

Keywords and phrases: asymmetric tolerances, capability indices, estimation.

\section{Introduction}

The capability indices of a process are used in industry in order to measure the capability of a process to produce according to some tolerances $[L ; U]$. For a target $T$ located at the mid-point $m$ of the tolerance interval, the most frequently used capability indices are

$$
\begin{gathered}
C_{p}=\frac{U-L}{6 \sigma}=\frac{d}{3 \sigma}, \\
C_{p k}=\frac{\min (U-\mu ; \mu-L)}{3 \sigma}=\frac{d-|\mu-m|}{3 \sigma}, \\
C_{p m}=\frac{U-L}{3 \sqrt{E\left[(X-T)^{2}\right]}}=\frac{d}{6 \sqrt{\sigma^{2}+(\mu-T)^{2}}}, \\
C_{p m k}=\frac{\min (U-\mu, \mu-L)}{3 \sqrt{E\left[(X-T)^{2}\right]}}=\frac{d-|\mu-m|}{3 \sqrt{\sigma^{2}+(\mu-T)^{2}}},
\end{gathered}
$$

where $d=(U-L) / 2$ is half of the length of the tolerance interval, $m=(U+L) / 2$ the midpoint of the tolerance interval, $\mu$ the process mean and $\sigma$ the process standard deviation under stationary controlled conditions, and $E[$.$] denotes "expected value". C_{p}$ represents the potential capability of the process, that is to say the maximum capability which can be obtained in the optimal conditions of fabrication when the mean $\mu$ is on the target $T=m$. $C_{p}$ is directly related to the expected percentage of non-conforming items when the studied characteristic of the process is normally distributed and $\mu=m$. However, after Taguchi's works, the concept of quality should not be exclusively focused on the probability of nonconformance. Keeping the process on target is also very important. The three other indices 
take into account this new aspect, in a different way. In this approach, Vännman (1995) generalizes the previous indices by proposing the index

$$
C_{p}(u, v)=\frac{d-u|\mu-T|}{3 \sqrt{\sigma^{2}+v(\mu-T)^{2}}} .
$$

However, the infinity of choices thus offered does not simplify the task of the user. This is the reason why Vännman studies the properties of the estimators of $C_{p}(u, v)$ and offers a limited choice of one or two pairs $(u, v)$ considering a certain number of criteria of optimality. For asymmetrical tolerances, that is to say when the target is not on the mid-point of the tolerance interval, Vännman (1997) suggests the generalization

$$
C_{p a}(u, v)=\frac{d-|\mu-m|-u|\mu-T|}{3 \sqrt{\sigma^{2}+v(\mu-T)^{2}}} .
$$

When $\mu$ moves away from $T$ towards the closest tolerance limit this index decreases faster than when it goes towards the farthest tolerance limit, which is an advantage since the index must respond faster to the deviations towards the wrong side. However $C_{p a}(u, v)$ is not always maximum in $T$ and shows a singularity when the mean moves away towards the farthest tolerance limit. The generalization $C_{p}^{\prime \prime}(u, v)$ suggested by Chen and Pearn (2001) in the case of asymmetrical tolerances is not hindered by these disadvantages:

$$
C_{p}^{\prime \prime}(u, v)=\frac{d^{*}-u A^{*}}{3 \sqrt{\sigma^{2}+v A^{2}}}, u \geq 0, v \geq 0,
$$

where $A=\max \left\{d(\mu-T) / D_{u}, d(T-\mu) / D_{l}\right\}, A^{*}=\max \left\{d^{*}(\mu-T) / D_{u}, d^{*}(T-\mu) / D_{l}\right\}$, $D_{u}=U-T, D_{l}=T-L$, and $d^{*}=\min \left\{D_{u}, D_{l}\right\}$.

In an article accepted in 1995, but which to our knowledge, has not been published yet, Chen and Pearn (2001) indicate that they obtain the properties of the estimator $\hat{C}_{p}^{\prime \prime}(u, v)$ of $C_{p}^{\prime \prime}(u, v)$. Nevertheless, the properties of $\hat{C}_{p}^{\prime \prime}(u, v)$ have been studied for the usual special cases, by Pearn and Chen (1998), as well as by Pearn, Lin and Chen (2004) for the index $C_{p k}^{\prime \prime}=C_{p}^{\prime \prime}(1,0)$, by Chen, Pearn, and Lin (1999) for the index $C_{p m}^{\prime \prime}=C_{p}^{\prime \prime}(0,1)$, and by Pearn, Lin, and Chen $(1999,2001)$ for the index $C_{p m k}^{\prime \prime}=C_{p}^{\prime \prime}(1,1)$.

To begin with, the objective of this paper is to give the formulas for the r-th moment of $\hat{C}_{p}^{\prime \prime}(u, v)$. This enables us in particular, to give for $\hat{C}_{p k}^{\prime \prime}$ identical expressions of the moments of order 1 and 2 , but simpler than Pearn's and Chen's (1998).

Then, following Vännman's approach (1995) concerning the symmetrical case, the properties of the estimators $\hat{C}_{p}^{\prime \prime}(u, v)$ will be used to choose the pairs $(u, v)$ which come up to criteria of optimality.

\section{Estimation of $C_{p}^{\prime \prime}(u, v)$}

The studied characteristic of the process is supposed normally distributed with mean $\mu$ and variance $\sigma^{2}$. Two natural estimators of $C_{p}^{\prime \prime}(u, v)$ can be considered, differing in the way the variance $\sigma^{2}$ is estimated. As Vännman (1995), we define the estimators $\hat{C}_{p, n}^{\prime \prime}(u, v)$ and $\hat{C}_{p, n-1}^{\prime \prime}(u, v)$ as 


$$
\hat{C}_{p, n}^{\prime \prime}(u, v)=\frac{d^{*}-u \hat{A}^{*}}{3 \sqrt{S_{n}^{2}+v \hat{A}^{2}}}, \text { and } \hat{C}_{p, n-1}^{\prime \prime}(u, v)=\frac{d^{*}-u \hat{A}^{*}}{3 \sqrt{S_{n-1}^{2}+v \hat{A}^{2}}},
$$

where $\hat{A}=\max \left\{d(\bar{X}-T) / D_{u}, d(T-\bar{X}) / D_{l}\right\}, \hat{A}^{*}=\max \left\{d^{*}(\bar{X}-T) / D_{u}, d^{*}(T-\bar{X}) / D_{l}\right\}$, $\bar{X}=\left(\sum_{i=1}^{n} X_{i}\right) / n, S_{n}^{2}=\sum_{i=1}^{n}\left(X_{i}-\bar{X}\right)^{2} / n$, and $S_{n-1}^{2}=\sum_{i=1}^{n}\left(X_{i}-\bar{X}\right)^{2} /(n-1)$.

Later on, we will consider only the estimator $\hat{C}_{p, n}^{\prime \prime}(u, v)$ since the two estimators are related by

$$
\hat{C}_{p, n-1}^{\prime \prime}(u, v)=\sqrt{\frac{n-1}{n}} \hat{C}_{p, n}^{\prime \prime}\left(u, \frac{n-1}{n} v\right) .
$$

By a demonstration identical to Pearn's, Lin's, and Chen's (1999), assuming that $\delta=\sqrt{n}(\mu-T) / \sigma, \lambda=\delta^{2}$, et $D^{*}=\sqrt{n} d^{*} / \sigma$, we obtain

$$
\begin{aligned}
& E\left(\hat{C}_{p, n}^{\prime \prime}(u, v)\right)^{r}=\frac{1}{3^{r}} \sum_{i=0}^{r}(-u)^{i}\left(\begin{array}{c}
r \\
i
\end{array}\right)\left(\frac{D^{*}}{\sqrt{2}}\right)^{r-i} \frac{e^{-\lambda / 2}}{2 \sqrt{\pi}} \times \sum_{j=0}^{\infty} \frac{\delta^{j} 2^{j / 2}}{j !} \frac{\Gamma((n-r+i+j) / 2) \Gamma((1+i+j) / 2)}{\Gamma((n+i+j) / 2)} \\
& \times\left[\left(\frac{d^{*}}{D_{u}}\right)^{i}{ }_{2} F_{1}\left(a, b ; c ; z_{u}\right)+(-1)^{j}\left(\frac{d^{*}}{D_{l}}\right)^{i}{ }_{2} F_{1}\left(a, b ; c ; z_{l}\right)\right], \quad(1)
\end{aligned}
$$

where $(-u)^{i}$ should be interpreted as 1 when $i=0$, also for the case $u=0$, and ${ }_{2} F_{1}(a, b ; c ; z)$ is the Gaussian hypergeometric function (Abramowitz and Stegun (1965)) with parameters $a=r / 2, b=(1+i+j) / 2, c=(n+i+j) / 2, z_{u}=1-\left(d / D_{u}\right)^{2} v$, and $z_{l}=1-\left(d / D_{l}\right)^{2} v$.

When the manufacturing tolerances are symmetric $(T=m)$, then $d^{*}=d=D_{u}=D_{l}$, $D^{*}=D=\sqrt{n} d / \sigma$, and the expression in (1) reduces to

$$
\begin{aligned}
E\left(\hat{C}_{p, n}^{\prime \prime}(u, v)\right)^{r}= & \frac{1}{3^{r}} \sum_{i=0}^{r}(-u)^{i}\left(\begin{array}{l}
r \\
i
\end{array}\right)\left(\frac{D}{\sqrt{2}}\right)^{r-i} e^{-\lambda / 2} \\
& \times \sum_{j=0}^{\infty} \frac{\delta^{2 j}}{2^{j} j !} \frac{\Gamma((n-r+i) / 2+j) \Gamma((1+i) / 2+j)}{\Gamma(1 / 2+j) \Gamma((n+i) / 2+j)}{ }_{2} F_{1}\left(\frac{r}{2}, \frac{1+i}{2}+j ; \frac{n+i}{2}+j ; 1-v\right),
\end{aligned}
$$

which is identical to that obtained in Vännman (1995) for the symmetrical case.

When the process is on target, that is $\mu=T$, which is equivalent to $\delta=0$, the expression in

(1) reduces to

$$
\begin{aligned}
E\left(\hat{C}_{p, n}^{n}(u, v)\right)^{r}=\frac{1}{3^{r}} \sum_{i=0}^{r}(-u)^{i}\left(\begin{array}{l}
r \\
i
\end{array}\right)\left(\frac{D^{*}}{\sqrt{2}}\right)^{r-i} \frac{1}{2 \sqrt{\pi}} \times \frac{\Gamma((n-r+i) / 2) \Gamma((1+i) / 2)}{\Gamma((n+i) / 2)} \\
\times\left[\left(\frac{d^{*}}{D_{u}}\right)^{i}{ }_{2} F_{1}\left(\frac{r}{2}, \frac{1+i}{2} ; \frac{n+i}{2} ; z_{u}\right)+\left(\frac{d^{*}}{D_{l}}\right)^{i}{ }_{2} F_{1}\left(\frac{r}{2}, \frac{1+i}{2} ; \frac{n+i}{2} ; z_{l}\right)\right] .
\end{aligned}
$$

In the special case when $u=0$, the expression in (1) reduces to

$$
\begin{aligned}
E\left(\hat{C}_{p, n}^{\prime \prime}(0, v)\right)^{r}=\frac{1}{3^{r}}\left(\frac{D^{*}}{\sqrt{2}}\right)^{r} \frac{e^{-\lambda / 2}}{2 \sqrt{\pi}} \times \sum_{j=0}^{\infty} & \frac{\delta^{j} 2^{j / 2}}{j !} \frac{\Gamma((n-r+j) / 2) \Gamma((1+j) / 2)}{\Gamma((n+j) / 2)} \\
& \times\left[{ }_{2} F_{1}\left(\frac{r}{2}, \frac{1+j}{2} ; \frac{n+j}{2} ; z_{u}\right)+(-1)^{j}{ }_{2} F_{1}\left(\frac{r}{2}, \frac{1+j}{2} ; \frac{n+j}{2} ; z_{l}\right)\right] .
\end{aligned}
$$

Furthermore, if $\mu=T$, 


$$
E\left(\hat{C}_{p, n}^{\prime \prime}(0, v)\right)^{r}=\frac{1}{3^{r}}\left(\frac{D^{*}}{\sqrt{2}}\right)^{r} \frac{1}{2} \times \frac{\Gamma((n-r) / 2)}{\Gamma(n / 2)}\left[{ }_{2} F_{1}\left(\frac{r}{2}, \frac{1}{2} ; \frac{n}{2} ; z_{u}\right)+{ }_{2} F_{1}\left(\frac{r}{2}, \frac{1}{2} ; \frac{n}{2} ; z_{l}\right)\right] .
$$

In the special case when $v=0$, the expression in (1) reduces to

$$
\begin{aligned}
E\left(\hat{C}_{p, n}^{\prime \prime}(u, 0)\right)^{r}=\frac{1}{3^{r}} \sum_{i=0}^{r}(-u)^{i}\left(\begin{array}{l}
r \\
i
\end{array}\right)\left(\frac{D^{*}}{\sqrt{2}}\right)^{r-i} & \frac{e^{-\lambda / 2}}{2 \sqrt{\pi}} \times \frac{\Gamma((n-r-1) / 2)}{\Gamma((n-1) / 2)} \\
& \times \sum_{j=0}^{\infty} \frac{\delta^{j} 2^{j / 2}}{j !} \Gamma((1+i+j) / 2)\left[\left(\frac{d^{*}}{D_{u}}\right)^{i}+(-1)^{j}\left(\frac{d^{*}}{D_{l}}\right)^{i}\right] .
\end{aligned}
$$

Furthermore, if $\mu=T$,

$$
E\left(\hat{C}_{p, n}^{n}(u, 0)\right)^{r}=\frac{1}{3^{r}} \sum_{i=0}^{r}(-u)^{i}\left(\begin{array}{l}
r \\
i
\end{array}\right)\left(\frac{D^{*}}{\sqrt{2}}\right)^{r-i} \frac{1}{2 \sqrt{\pi}} \times \frac{\Gamma((n-r-1) / 2) \Gamma((1+i) / 2)}{\Gamma((n-1) / 2)}\left[\left(\frac{d^{*}}{D_{u}}\right)^{i}+\left(\frac{d^{*}}{D_{l}}\right)^{i}\right] .
$$

In the special case when $(u, v)=(0,0)$, using lemma 1 of appendix 1 ,

$$
E\left(\hat{C}_{p, n}^{\prime \prime}(0,0)\right)^{r}=\left(\frac{n}{2}\right)^{r / 2} \frac{\Gamma((n-r-1) / 2)}{\Gamma((n-1) / 2)} C_{p}^{\prime \prime}(0,0)^{r} .
$$

When $(u, v)=(0,1)$ we obtain the expressions given by Chen, Pearn, and Lin (1999) for $C_{p m}^{\prime \prime}=C_{p}^{\prime \prime}(0,1)$. When $(u, v)=(1,1)$ we obtain the expressions given by Pearn, Lin, and Chen (1999) for $C_{p m k}^{\prime \prime}=C_{p}^{\prime \prime}(1,1)$. When $(u, v)=(1,0)$, the equation (2) allows to obtain the first two moments in the following form :

$$
\begin{aligned}
& E\left(\hat{C}_{p, n}^{\prime \prime}(1,0)\right)=\sqrt{\frac{n}{2}} \frac{\Gamma((n-2) / 2)}{\Gamma((n-1) / 2)}\left[C_{p}^{\prime \prime}(1,0)+\frac{1}{3 \sqrt{n}}\left(\frac{d^{*}}{D_{u}}+\frac{d^{*}}{D_{l}}\right)\left(|\delta| \Phi(-|\delta|)-\frac{e^{-\delta^{2} / 2}}{\sqrt{2 \pi}}\right)\right], \\
& E\left(\hat{C}_{p, n}^{\prime \prime}(1,0)\right)^{2}=\frac{n}{(n-3)}\left[C_{p}^{\prime \prime}(1,0)^{2}-\frac{2}{9 \sqrt{n}} \frac{d^{*}}{\sigma}\left(\frac{d^{*}}{D_{u}}+\frac{d^{*}}{D_{l}}\right)\left(\frac{e^{-\delta^{2} / 2}}{\sqrt{2 \pi}}-|\delta| \Phi(-|\delta|)\right)\right. \\
& \left.+\frac{1}{18 n}\left(\left(\frac{d^{*}}{D_{u}}\right)^{2}+\left(\frac{d^{*}}{D_{l}}\right)^{2}\right)+\frac{\delta}{9 n}\left(\left(\frac{d^{*}}{D_{u}}\right)^{2}-\left(\frac{d^{*}}{D_{l}}\right)^{2}\right)\left(\frac{e^{-\delta^{2} / 2}}{\sqrt{2 \pi}}-|\delta| \Phi(-|\delta|)+\frac{|\delta|}{2 \delta^{2}}\{1-2 \Phi(-|\delta|)\}\right)\right] .
\end{aligned}
$$

These expressions are identical but simpler (see appendix 1) that those given by Pearn and Chen (1998), except for a coefficient since they calculate $E\left(\hat{C}_{p, n-1}^{\prime \prime}(1,0)\right)$ and $E\left(\hat{C}_{p, n-1}^{\prime \prime}(1,0)\right)^{2}$.

\section{Optimality Criteria}

Here we take up with slight modifications, the approach suggested by Vänmann (1995) for symmetrical tolerances, which we extend to the case of asymmetrical tolerances. Several studies are carried out according to the position of the target within the tolerance interval. This position is located by the parameter $d / D_{u}$ which will be studied for the values $1,4 / 3,2$, and 4 . When $d / D_{u}=1$ the target is located at the midpoint of the tolerance interval, which is the symmetrical case already studied by Vänmann (1995). For the values $d / D_{u}=$ $4 / 3,2,4$, the target is located at the quarter of the interval $[m ; U]$ starting from $m$, at the midpoint of the interval $[m ; U]$, and at the three-quarters of the interval $[m ; U]$ starting from $m$. Of course the results are identical and will thus not be studied for a target located on the side of the lower limit $L$. One of the purposes of Vänmann was to find an index sensitive to 
deviations from the target value, especially in the case when $\sigma$ is small, because in such a case it is more difficult to detect the fact that the process is not capable as it is not on the target. Since $C_{p}^{\prime \prime}(u, v)=\left(1-u A^{*} / d^{*}\right)\left(1+v A^{2} / \sigma^{2}\right)^{-1 / 2} C_{p}^{\prime \prime}(0,0)$, for any value of $\mu$ within the tolerance interval, we have $C_{p}^{\prime \prime}(u+x, v+y) \leq C_{p}^{\prime \prime}(u, v)$, for any $x, y \geq 0$. This shows that if we want the index to be sensitive to deviations of $\mu$ from $T$, then $u$ and $v$ should be large. Of course, the index being unknown, we wish its estimator to be sensitive. However it would be useless to have a very sensitive estimator if it was not reliable, that is to say if its bias and its mean square error (MSE) were important. Various calculations have been carried out varying the parameters $a=(\mu-T) / \sigma=0,2.0(0.5), b=d^{*} / \sigma=2,6(1), n=10,50(10), u=0,5(1)$, and $v$ $=0,5(1)$. Only integer values of $u$ and $v$ were utilized since it seems not to be of any interest to consider more complicated indices The results show that in general, the larger $u$ and $v$ are the more sensitive the estimator is, but also the larger the bias and the MSE are. However this is not always true when $a$ is equal to 0 or close to 0 . For example, for $b=2, n=10, a=0$, the bias and the MSE of $\hat{C}_{p, n}^{\prime \prime}(0,1)$ are equal to 0.084 and 0.037 , whereas those of $\hat{C}_{p, n}^{\prime \prime}(0,5)$ are equal to -0.035 and 0.034 . For $b=2, n=10, a=0.1$, the bias and the MSE of $\hat{C}_{p, n}^{\prime \prime}(0,1)$ are equal to 0.056 and 0.036 , whereas those of $\hat{C}_{p, n}^{\prime \prime}(0,5)$ are equal to -0.026 and 0.034 . We thus should find a compromise between the quality and the sensitivity of the estimator. Hence the following strategy :

Step 1:

When $a=0$, choosing the values of $u$ and $v$ that will give simultaneously small values of the relative bias and the mean square error.

It is the most important property, for a reliable estimator is essential when the process is working in optimal conditions. In practice, we calculate the relative bias and the MSE for the various values of $b$ and $n$, which gives us 25 possible cases. We keep the 4 pairs $(u, v)$ which are simultaneously the best, in these 25 situations.

Step 2 :

Selecting the values of $u$ and $v$ for which the estimator of $C_{p}^{\prime \prime}(u, v)$ has an expected value that is sensitive to departures from $T$.

An estimator is all the more sensitive to the deviations of $\mu$ from $T$, as its expected value decreases quickly when $\mu$ moves away from $T$. In order to measure the sensitivity of the estimator $\hat{C}_{p, n}^{\prime \prime}(u, v)$, we use the quantity called $S$ equal to the quotient of $E\left(\hat{C}_{p, n}^{\prime \prime}(u, v)\right)$ when $a \neq 0$ by $E\left(\hat{C}_{p, n}^{\prime \prime}(u, v)\right)$ when $a=0$. By way of comparison, we calculate the sensitivity of the index $C_{p}^{\prime \prime}(u, v)$, also called $S$, equal to the quotient of $C_{p}^{\prime \prime}(u, v)$ when $a \neq 0$ by $C_{p}^{\prime \prime}(u, v)$ when $a$ $=0$, that is to say the quotient of $C_{p}^{\prime \prime}(u, v)$ when $a \neq 0$ by $C_{p}^{\prime \prime}(0,0)$. The smaller $S$ is, the more the expected value of the estimator decreases, and then the more sensitive the estimator is. The expressions of $E\left(\hat{C}_{p, n}^{\prime \prime}(0, v)\right)$ and $C_{p}^{\prime \prime}(0, v)$ allow to note that the sensitivity is independent of $b$ when $u=0$, and thus depends only on $a$ and $n$ in that case.

Unfortunately an index cannot have the best sensitivity for any value of $a, b$, and $n$. As we need an index, especially sensitive when $\sigma$ is small, we will first examine the greatest values of $b$. If necessary, we will compare then the sensitivity for the lowest values of $|a|$, because the index must quickly detect the deviations of the mean. In practice we will eliminate the least sensitive pair and keep only three for the next step.

Step 3 : 
Choosing an index according to the quality of the estimators for any $a$, and to the sensitivity, and the facility of interpretation for the practitioner.

At the previous steps, the indices were kept for their low values of relative bias and MSE when $a=0$. It is now necessary to compare them for any value of $a$. For indices having rather similar properties, the practitioner will no doubt prefer indices he is able to interpret, even if that means a slight loss in the quality of the estimators and the sensitivity. Whatever the position of the mean within the tolerance interval, we always have $C_{p}^{\prime \prime}(u, v) \leq C_{p}^{\prime \prime}(0,0)$, equality happening only when the mean is on the target. Thus, $C_{p}^{\prime \prime}(0,0)$ represents the potential capability, that is to say the maximum capability to be hoped for in the optimal conditions of production. When the mean moves away from the target and reaches the tolerance bounds, we have $C_{p}^{\prime \prime}(u, v)=d^{*}(1-u) /\left(3 \sqrt{\sigma^{2}+v d^{2}}\right)$, which is thus positive if $u=0$, null if $u=1$, negative if $u>1$. Of course the case $u=1$ provides the easiest interpretation because the index varies between $C_{p}^{\prime \prime}(0,0)$ when the mean is on the target and 0 when the mean reaches the interval bounds. The case $u>1$ is the least interesting because the index can take negative values within the tolerance interval. The interpretation is made easier since it is linked to $u$, with $u=1, u=0$, then $u>1$ in order of preference.

In the next paragraphs, we give the results of each step for various positions of the target. All the calculations which have been carried out cannot be given here. Only some tables of results allowing the reader to follow the adopted strategy are given in appendix 2 .

\section{Optimal indices when $d / D_{u}=1$}

\section{Step 1:}

We are in the situation where $a=0$. As an example, consider the case when $n=30$. When $b=$ 2 and 6 , Table 1 gives the relative bias of $\hat{C}_{p, n}^{\prime \prime}(u, v)$, and Table 2 gives the mean square error MSE of $\hat{C}_{p, n}^{\prime \prime}(u, v)$ multiplied by 100. If in each one of these 4 tables we choose the 8 best pairs, the common pairs selected are $(u, v)=(0,2),(0,3),(0,4),(1,0),(1,1),(1,2)$. For the values $n=10,50(10)$ and $b=2,6(1)$, we obtain 25 tables which are not reproduced here. By selecting the 8 best pairs out of these 25 tables, the common couples selected are $(u, v)=(0,2)$, $(0,3),(0,4),(1,2)$.

Step 2 :

For example, table 3 gives the sensitivity of the estimator $\hat{C}_{p, n}^{\prime \prime}(u, v)$ and of the index $C_{p}^{\prime \prime}(u, v)$ for the previous pairs when $n=10,30,50$, and $b=2,6$. We note that the least sensitive is $\hat{C}_{p, n}^{\prime \prime}(0,2)$, then $\hat{C}_{p, n}^{\prime \prime}(0,3)$. For $\hat{C}_{p, n}^{\prime \prime}(0,4)$ and $\hat{C}_{p, n}^{\prime \prime}(1,2)$ the situation is less clear. So, we compare them for $b=6$. In that case again, the comparison is not obvious. Then we restrict ourselves to the small values of $a$, and reach the conclusion that $\hat{C}_{p, n}^{\prime \prime}(0,4)$ is the most sensitive. The study for the other values of $n$, not given here, leads to the same conclusions. At this stage we retain only the pairs $(u, v)=(0,3),(0,4),(1,2)$.

Step 3 :

For these 3 pairs Table 4 gives the relative bias of $\hat{C}_{p, n}^{\prime \prime}(u, v)$ when $n=30, b=2,6$ and Table 5 gives the MSE of $\hat{C}_{p, n}^{\prime \prime}(u, v)$ multiplied by 100 when $n=30, b=2,6(1)$. We note that $\hat{C}_{p, n}^{\prime \prime}(0,3)$ and $\hat{C}_{p, n}^{\prime \prime}(0,4)$ are very similar and slightly better than $\hat{C}_{p, n}^{\prime \prime}(1,2)$. All the results which are not given here lead to the same conclusions. In conclusion, as Vännman (1995) 
shows it, if we had to retain one index, the choice would be $C_{p}^{\prime \prime}(0,3)$ if the quality of the estimator is to be privileged because it is almost unbiased when the process is on target, while $C_{p}^{\prime \prime}(0,4)$ and $C_{p}^{\prime \prime}(1,2)$ have a small but negative bias. If sensitivity is the main criterion to be retained, we will select $C_{p}^{\prime \prime}(0,4)$. The facility of interpretation, which seems an important criterion for the practitioner is not taken into account by Vännman. If this criterion is chosen, we recommend to use $C_{p}^{\prime \prime}(1,2)$.

\section{Optimal indices when $d / D_{u}=4 / 3$}

\section{Step 1 :}

$a=0$. When $n=30, b=2,6$, Table 6 gives the relative bias of $\hat{C}_{p, n}^{\prime \prime}(u, v)$, and Table 7 gives the MSE of $\hat{C}_{p, n}^{\prime \prime}(u, v)$ multiplied by 100 . If in each one of these 4 tables we choose the 10 best pairs, the common pairs selected are $(u, v)=(0,1),(0,2),(0,3),(0,4),(1,0),(1,1),(1,2)$, $(2,0)$. For the other values of $n$ and $b$ not given here, the 4 best common pairs are obtained from the 10 best pairs of each table, and are $(u, v)=(0,2),(0,3),(0,4),(1,2)$.

\section{Step 2 :}

Table 8 gives the sensitivity of the estimator $\hat{C}_{p, n}^{\prime \prime}(u, v)$ and of the index $C_{p}^{\prime \prime}(u, v)$ for the previous pairs when $n=10,30,50$, and $b=2,6$. We note that the least sensitive is $\hat{C}_{p, n}^{\prime \prime}(0,2)$. We compare the 3 others for $b=6$ and the small values of $|a|$. We note that the most sensitive estimator is $\hat{C}_{p, n}^{\prime \prime}(0,4)$, then $\hat{C}_{p, n}^{\prime \prime}(0,3)$, and finally $\hat{C}_{p, n}^{\prime \prime}(1,2)$. The study for the other values of $n$, not given here, leads to the same conclusions. At this stage we retain only the pairs $(u, v)$ $=(0,3),(0,4),(1,2)$.

\section{Step 3 :}

For these 3 pairs Table 9 gives the relative bias of $\hat{C}_{p, n}^{\prime \prime}(u, v)$ when $n=30, b=2,6$ and Table 10 gives the MSE of $\hat{C}_{p, n}^{\prime \prime}(u, v)$ multiplied by 100 when $n=30, b=2,6(1)$. We note that $\hat{C}_{p, n}^{\prime \prime}(0,3)$ and $\hat{C}_{p, n}^{\prime \prime}(0,4)$ are very similar and slightly better than $\hat{C}_{p, n}^{\prime \prime}(1,2)$ with regard to the relative bias. As regards the MSE it is difficult to make a distinction between the 3 estimators. All the results which are not given here lead to the same conclusions. As a conclusion for the case $d / D u=4 / 3$, if we must retain one index, the choice could be done according to the criterion to which preference is given:

If the quality of the estimators is chosen : retain $C_{p}^{\prime \prime}(0,3)$ which is the least biased when the process is on target.

If it is the sensitivity : retain $C_{p}^{\prime \prime}(0,4)$.

If it is the facility of interpretation : retain $C_{p}^{\prime \prime}(1,2)$.

\section{Optimal indices when $d / D_{u}=2$}

\section{Step 1 :}

$a=0$. When $n=30, b=2,6$, Table 11 gives the relative bias of $\hat{C}_{p, n}^{\prime \prime}(u, v)$, and Table 12 gives the MSE of $\hat{C}_{p, n}^{\prime \prime}(u, v)$ multiplied by 100. If in each one of these 4 tables we choose the 10 best pairs, the common pairs selected are $(u, v)=(0,1),(0,2),(1,0),(1,1),(1,2),(2,0)$. For the 
other values of $n$ and $b$ not given here, the 4 best common pairs are obtained from the 10 best pairs of each table, and are $(u, v)=(0,1),(0,2),(1,1),(1,2)$.

\section{Step 2 :}

Table 13 gives the sensitivity of the estimator $\hat{C}_{p, n}^{\prime \prime}(u, v)$ and of the index $C_{p}^{\prime \prime}(u, v)$ for the previous pairs when $n=10,30,50$, and $b=2,6$. We note that $\hat{C}_{p, n}^{n}(1,2)$ is the most sensitive and $\hat{C}_{p, n}^{\prime \prime}(0,1)$ the least sensitive. We compare the 2 others for $b=6$ and the small values of $|a|$. We note that the most sensitive estimator is $\hat{C}_{p, n}^{\prime \prime}(0,2)$, then $\hat{C}_{p, n}^{\prime \prime}(1,1)$. The study for the other values of $n$, not given here, leads to the same conclusions. At this stage we retain only the pairs $(u, v)=(0,2),(1,1),(1,2)$.

Step 3 :

For these 3 pairs Table 14 gives the relative bias of $\hat{C}_{p, n}^{\prime \prime}(u, v)$ when $n=30, b=2,6$ and Table 15 gives the MSE of $\hat{C}_{p, n}^{\prime \prime}(u, v)$ multiplied by 100 when $n=30, b=2,6(1)$. These two tables, as well as those made for the other values of the parameters do not allow to distinguish one estimator from the other 2 .

In conclusion, for $d / D_{u}=2$, we recommend to use $\hat{C}_{p, n}^{\prime \prime}(1,2)$ which is the most sensitive, and the interpretation of which is easy.

\section{Optimal indices when $d / D_{u}=4$}

\section{Step 1 :}

$a=0$. When $n=30, b=2,6$, Table 16 gives the relative bias of $\hat{C}_{p, n}^{\prime \prime}(u, v)$, and Table 17 gives the MSE of $\hat{C}_{p, n}^{\prime \prime}(u, v)$ multiplied by 100. If in each one of these 4 tables we choose the 7 best pairs, the common pairs selected are $(u, v)=(0,1),(1,0),(1,1),(2,0),(3,0)$. For the other values of $n$ and $b$ not given here, the 4 best common pairs are obtained from the 7 best pairs of each table, and are $(u, v)=(0,1),(1,1),(2,0),(3,0)$.

Step 2 :

Table 18 gives the sensitivity of the estimator $\hat{C}_{p, n}^{\prime \prime}(u, v)$ and of the index $C_{p}^{\prime \prime}(u, v)$ for the previous pairs when $n=10,30,50$, and $b=2,6$. As we have already noted, $C_{p}^{\prime \prime}(u, 0)$ and consequently its expected value become negative when the mean approaches the tolerance bounds. As it is not very judicious to measure the capability using a negative quantity, we did not copy out such values on table 18 . No pair, even for $b=6$ and $a=-0.5,0.5$ allows any conclusion. Therefore in table 18 we have added the values of the sensitivity for values of the mean nearer to the target, considering $a=-0.1$ and 0.1 . As can be noted, the rank is completely reversed according to whether the mean moves towards the lower or the upper limit. It is then impossible to draw any conclusion concerning the sensitivity of these 4 estimators.

\section{Step 3 :}

For these 4 previous pairs, Table 19 gives the relative bias of $\hat{C}_{p, n}^{\prime \prime}(u, v)$ when $n=30, b=2,6$ and Table 20 gives the MSE of $\hat{C}_{p, n}^{\prime \prime}(u, v)$ multiplied by 100 when $n=30, b=2,6(1)$. As in the previous case, the negative values have not been copied out on these tables. These two tables, as those carried out for the other values of the parameters, allow to note that $C_{p}^{\prime \prime}(0,1)$ and $C_{p}^{\prime \prime}(1,1)$ on the one hand, $C_{p}^{\prime \prime}(2,0)$ and $C_{p}^{\prime \prime}(3,0)$ on the other hand, are very similar, but 
none can be considered as better than the others. Under these conditions, since none of the estimators is preferable for its sensitivity or its reliability, only the criterion of interpretation is retained. This is the reason why we recommend the use of $C_{p}^{\prime \prime}(1,1)$ for $d / D_{u}=4$.

\section{Appendix 1}

In order to obtain $E\left(\hat{C}_{p, n}^{\prime \prime}(u, 0)\right)$ and $E\left(\hat{C}_{p, n}^{\prime \prime}(u, 0)\right)^{2}$, the following preliminary results are used:

Lemma $1: \sum_{j=0}^{\infty} \frac{\delta^{j}\left(1+(-1)^{j}\right)}{2^{j / 2} \Gamma(j / 2+1)}=2 e^{\delta^{2} / 2}$

Lemma $2: \sum_{j=0}^{\infty} \frac{\delta^{2 j}}{2^{j} \Gamma(j+1 / 2)}=\frac{1}{\sqrt{\pi}}+e^{\delta^{2} / 2} \frac{|\delta|}{\sqrt{2}}[1-2 \Phi(-|\delta|)]$

Indeed, if we assume that $\mathfrak{L}$ is the Laplace transformation,

$$
\begin{aligned}
& \\
& \qquad\left(\sum_{j=0}^{\infty} \frac{(\lambda / 2)^{j}}{\Gamma(j+1 / 2)}\right)=\sqrt{\lambda} \mathfrak{L}\left(\sum_{j=0}^{\infty} \frac{\lambda^{j-1 / 2}}{2^{j} \Gamma(j+1 / 2)}\right)=\sqrt{\lambda} \sum_{j=0}^{\infty} \frac{1}{2^{j} p^{j+1 / 2}}=\sqrt{\lambda}\left(\frac{1}{\sqrt{p}}+\frac{1}{2 \sqrt{p}(p-1 / 2)}\right) \\
& =\sqrt{\lambda}\left[\mathfrak{L}\left(\frac{1}{\sqrt{\pi \lambda}}\right)+\frac{1}{2} \mathfrak{L}\left(\frac{e^{\lambda / 2}}{\sqrt{1 / 2}} \operatorname{erf}(\sqrt{\lambda / 2})\right]=\mathfrak{L}\left(\frac{1}{\sqrt{\pi}}+2 e^{\lambda / 2} \sqrt{\frac{\lambda}{2}}[\Phi(\sqrt{\lambda})-1 / 2]\right)\right. \\
& =\mathfrak{L}\left(\frac{1}{\sqrt{\pi}}+e^{\lambda / 2} \sqrt{\frac{\lambda}{2}}[1-2 \Phi(-\sqrt{\lambda})]\right), \text { hence the lemma since } \lambda=\delta^{2} .
\end{aligned}
$$

Lemma $3: \sum_{j=0}^{\infty} \frac{\delta^{2 j+1}}{2^{j+1 / 2} \Gamma(j+1)}=\frac{\delta}{\sqrt{2}} e^{\delta^{2} / 2}$

Lemma $4: \sum_{j=0}^{\infty} \frac{(2 j+1) \delta^{2 j}}{2^{j+1} \Gamma(j+1)}=\frac{e^{\delta^{2} / 2}}{2}\left(1+\delta^{2}\right)$

Lemma $5: \sum_{j=0}^{\infty} \frac{(2 j+2) \delta^{2 j+1}}{2^{j+3 / 2} \Gamma(j+3 / 2)}=\frac{\delta}{\sqrt{2 \pi}}+\operatorname{sgn}(\delta) \frac{e^{\delta^{2} / 2}}{2}\left(1+\delta^{2}\right)[1-2 \Phi(-|\delta|)]$

Indeed, $\sum_{j=0}^{\infty} \frac{(2 j+2) \delta^{2 j+1}}{2^{j+3 / 2} \Gamma(j+3 / 2)}=\frac{1}{\sqrt{2}} \frac{d}{d \delta}\left(\sum_{j=0}^{\infty} \frac{\delta^{2 j}}{2^{j} \Gamma(j+1 / 2)}\right)$, hence the result according to lemma 2.

$$
E\left(\hat{C}_{p, n}^{n}(u, 0)\right) \text { is obtained from the equation (2), by using the formulas of duplication }
$$

of the function $\Gamma$,

$$
\begin{aligned}
E\left(\hat{C}_{p, n}^{n}(u, 0)\right)=\frac{1}{3} \frac{\Gamma((n-2) / 2)}{\Gamma((n-1) / 2)}\left[\frac{D^{*}}{\sqrt{2}} \frac{e^{-\lambda / 2}}{2} \sum_{j=0}^{\infty} \frac{\delta^{j}\left(1+(-1)^{j}\right)}{2^{j / 2} \Gamma(j / 2+1)}\right. & \left.-u \frac{e^{-\lambda / 2}}{2} \sum_{j=0}^{\infty} \frac{\delta^{j}}{2^{j / 2} \Gamma((j+1) / 2)}\left(\frac{d^{*}}{D_{u}}+(-1)^{j} \frac{d^{*}}{D_{l}}\right)\right],
\end{aligned}
$$

then by using lemmas 1,2 , and 3 , 


$$
\begin{aligned}
& E\left(\hat{C}_{p, n}^{\prime \prime}(u, 0)\right)=\frac{1}{3} \frac{\Gamma((n-2) / 2)}{\Gamma((n-1) / 2)}\left[\frac{D^{*}}{\sqrt{2}}-u \frac{e^{-\delta^{2} / 2}}{2}\right. \\
& \left.\quad \times\left\{\left(\frac{1}{\sqrt{\pi}}+e^{\delta^{2} / 2} \frac{|\delta|}{\sqrt{2}}[1-2 \Phi(-|\delta|)]\right)\left(\frac{d^{*}}{D_{u}}+\frac{d^{*}}{D_{l}}\right)+\frac{\delta}{\sqrt{2}} e^{\delta^{2} / 2}\left(\frac{d^{*}}{D_{u}}-\frac{d^{*}}{D_{l}}\right)\right\}\right] \\
& =\frac{1}{3} \frac{\Gamma((n-2) / 2)}{\Gamma((n-1) / 2)}\left[\frac{D^{*}}{\sqrt{2}}-\frac{u}{2}\left\{\left(\frac{d^{*}}{D_{u}}+\frac{d^{*}}{D_{l}}\right)\left(\frac{e^{-\delta^{2} / 2}}{\sqrt{\pi}}-\sqrt{2}|\delta| \Phi(-|\delta|)\right)+\frac{\sqrt{2}}{\sigma} \sqrt{n} A^{*}\right\}\right] \\
& =\sqrt{\frac{n}{2}} \frac{\Gamma((n-2) / 2)}{\Gamma((n-1) / 2)}\left[C_{p}^{\prime \prime}(u, 0)+\frac{u}{3 \sqrt{n}}\left(\frac{d^{*}}{D_{u}}+\frac{d^{*}}{D_{l}}\right)\left(|\delta| \Phi(-|\delta|)-\frac{e^{-\delta^{2} / 2}}{\sqrt{2 \pi}}\right)\right] .
\end{aligned}
$$

$E\left(\hat{C}_{p, n}^{n}(u, 0)\right)^{2}$ is obtained from the equation (2), by using the formulas of duplication of the function $\Gamma$,

$$
\begin{aligned}
E\left(\hat{C}_{p, n}^{\prime \prime}(u, 0)\right)^{2}=\frac{2}{9(n-3)}\left[\left(\frac{D^{*}}{\sqrt{2}}\right)^{2} \frac{e^{-\delta^{2} / 2}}{2} \sum_{j=0}^{\infty}\right. & \frac{\delta^{j}\left(1+(-1)^{j}\right)}{2^{j / 2} \Gamma(j / 2+1)} \\
& -u \frac{D^{*}}{\sqrt{2}} e^{-\delta^{2} / 2} \sum_{j=0}^{\infty} \frac{\delta^{j}}{2^{j / 2} \Gamma((j+1) / 2)}\left(\frac{d^{*}}{D_{u}}+(-1)^{j} \frac{d^{*}}{D_{l}}\right) \\
& \left.+u^{2} \frac{e^{-\delta^{2} / 2}}{2} \sum_{j=0}^{\infty} \frac{(j+1) \delta^{j}}{2^{j / 2+1} \Gamma(j / 2+1)}\left(\left(\frac{d^{*}}{D_{u}}\right)^{2}+(-1)^{j}\left(\frac{d^{*}}{D_{l}}\right)^{2}\right)\right] .
\end{aligned}
$$

Then by using lemmas 1,2, and 3, 4, and 5,

$$
\begin{aligned}
& E\left(\hat{C}_{p, n}^{\prime \prime}(u, 0)\right)^{2}=\frac{2}{9(n-3)}\left[\left(\frac{D^{*}}{\sqrt{2}}\right)^{2}-u \frac{D^{*}}{\sqrt{2}}\left\{\left(\frac{d^{*}}{D_{u}}+\frac{d^{*}}{D_{l}}\right)\left(\frac{e^{-\delta^{2} / 2}}{\sqrt{\pi}}-\sqrt{2}|\delta| \Phi(-|\delta|)\right)+\frac{\sqrt{2}}{\sigma} \sqrt{n} A^{*}\right\}\right. \\
& +u^{2} \frac{e^{-\delta^{2} / 2}}{2}\left\{\frac{e^{\delta^{2} / 2}}{2}\left(1+\delta^{2}\right)\left(\left(\frac{d^{*}}{D_{u}}\right)^{2}+\left(\frac{d^{*}}{D_{l}}\right)^{2}\right)\right\} \\
& \left.+\left(\frac{\delta}{\sqrt{2 \pi}}+\operatorname{sgn}(\delta) \frac{e^{\delta^{2} / 2}}{2}\left(1+\delta^{2}\right)\{1-2 \Phi(-|\delta|)\}\right)\left(\left(\frac{d^{*}}{D_{u}}\right)^{2}-\left(\frac{d^{*}}{D_{l}}\right)^{2}\right)\right] \\
& =\frac{n}{(n-3)}\left[C_{p}^{\prime \prime}(u, 0)^{2}-u \frac{2}{9 \sqrt{n}} \frac{d^{*}}{\sigma}\left(\frac{d^{*}}{D_{u}}+\frac{d^{*}}{D_{l}}\right)\left(\frac{e^{-\delta^{2} / 2}}{\sqrt{2 \pi}}-|\delta| \Phi(-|\delta|)\right)+u^{2} \frac{1}{18 n}\left(\left(\frac{d^{*}}{D_{u}}\right)^{2}+\left(\frac{d^{*}}{D_{l}}\right)^{2}\right)\right. \\
& \left.+u^{2} \frac{\delta}{9 n}\left(\left(\frac{d^{*}}{D_{u}}\right)^{2}-\left(\frac{d^{*}}{D_{l}}\right)^{2}\right)\left(\frac{e^{-\delta^{2} / 2}}{\sqrt{2 \pi}}-|\delta| \Phi(-|\delta|)+\frac{|\delta|}{2 \delta^{2}}\{1-2 \Phi(-|\delta|)\}\right)\right] .
\end{aligned}
$$




\section{Appendix 2}

Table $1: d / D_{u}=1$. Relative bias of $\hat{C}_{p, n}^{\prime \prime}(u, v)$ when the process is on target and $n=30$.

\begin{tabular}{|c|c|c|c|c|c|c|}
\hline$b=2$ & \multicolumn{7}{|c|}{$v$} \\
\hline$u$ & 0 & 1 & 2 & 3 & 4 & 5 \\
\hline 0 & & 0.026 & 0.009 & -0.004 & -0.017 & -0.029 \\
\hline 1 & -0.032 & -0.048 & -0.062 & -0.074 & -0.085 & -0.095 \\
\hline 2 & -0.108 & -0.121 & -0.133 & -0.143 & -0.153 & -0.162 \\
\hline 3 & -0.184 & -0.194 & -0.204 & -0.213 & -0.220 & -0.228 \\
\hline 4 & -0.260 & -0.268 & -0.276 & -0.282 & -0.288 & -0.294 \\
\hline 5 & -0.336 & -0.341 & -0.347 & -0.351 & -0.356 & -0.360 \\
\hline
\end{tabular}

\begin{tabular}{|c|c|c|c|c|c|c|}
\hline $\mathrm{b}=6$ & \multicolumn{6}{|c|}{$\mathrm{v}$} \\
\hline $\mathrm{u}$ & 0 & 1 & 2 & 3 & 4 & 5 \\
\hline 0 & & 0.026 & 0.009 & -0.004 & -0.017 & -0.029 \\
\hline 1 & 0.019 & 0.001 & -0.015 & -0.028 & -0.040 & -0.051 \\
\hline 2 & -0.006 & -0.023 & -0.039 & -0.051 & -0.062 & -0.073 \\
\hline 3 & -0.032 & -0.048 & -0.062 & -0.074 & -0.085 & -0.095 \\
\hline 4 & -0.057 & -0.072 & -0.086 & -0.097 & -0.108 & -0.117 \\
\hline 5 & -0.082 & -0.097 & -0.110 & -0.120 & -0.130 & -0.140 \\
\hline
\end{tabular}

Table 2: $d / D_{u}=1.100 \times \operatorname{MSE}$ of $\hat{C}_{p, n}^{\prime \prime}(u, v)$ when the process is on target and $n=30$.

\begin{tabular}{|c|c|c|c|c|c|c|}
\hline$b=2$ & \multicolumn{7}{|c|}{$v$} \\
\hline$u$ & 0 & 1 & 2 & 3 & 4 & 5 \\
\hline 0 & & 0.872 & 0.805 & 0.857 & 0.904 & 0.972 \\
\hline 1 & 0.973 & 1.074 & 1.193 & 1.348 & 1.494 & 1.654 \\
\hline 2 & 1.775 & 2.016 & 2.252 & 2.472 & 2.687 & 2.894 \\
\hline 3 & 3.401 & 3.699 & 3.983 & 4.227 & 4.466 & 4.692 \\
\hline 4 & 5.850 & 6.122 & 6.385 & 6.614 & 6.838 & 7.049 \\
\hline 5 & 9.121 & 9.286 & 9.458 & 9.633 & 9.801 & 9.965 \\
\hline
\end{tabular}

\begin{tabular}{|c|c|c|c|c|c|c|}
\hline$b=6$ & \multicolumn{6}{|c|}{$v$} \\
\hline$u$ & 0 & 1 & 2 & 3 & 4 & 5 \\
\hline 0 & & 7.852 & 7.558 & 7.709 & 8.138 & 8.752 \\
\hline 1 & 8.056 & 7.715 & 7.738 & 8.553 & 9.332 & 10.237 \\
\hline 2 & 7.995 & 8.319 & 8.902 & 10.028 & 11.117 & 12.281 \\
\hline 3 & 8.756 & 9.663 & 10.737 & 12.135 & 13.495 & 14.883 \\
\hline 4 & 10.341 & 11.749 & 13.244 & 14.873 & 16.465 & 18.044 \\
\hline 5 & 12.748 & 14.575 & 16.422 & 18.244 & 20.027 & 21.764 \\
\hline
\end{tabular}


Table 3: $d / D_{u}=1$. Sensitivity of $\hat{C}_{p, n}^{\prime \prime}(u, v)$ and $C_{p}^{\prime \prime}(u, v)$, when $(u, v)=(0,2),(0,3),(0,4)$, $(1,2)$ (top to bottom), and when $b=2,6$ (left to right)

\begin{tabular}{|c|cc|cc|cc|cc|}
\hline \multirow{7}{|c|}{ Sensitivity of $\hat{C}_{p, n}^{\prime \prime}(u, v)$} & & $\begin{array}{c}\text { Sensitivity of } \\
C_{p}^{\prime \prime}(u, v)\end{array}$ \\
\hline$a$ & $n=10$ & $n=30$ & $n=50$ & & \\
\hline \multirow{5}{*}{0.5} & 0.842 & 0.842 & 0.827 & 0.827 & 0.823 & 0.823 & 0.816 & 0.816 \\
& 0.809 & 0.809 & 0.778 & 0.778 & 0.770 & 0.770 & 0.756 & 0.756 \\
& 0.784 & 0.784 & 0.730 & 0.730 & 0.729 & 0.729 & 0.707 & 0.707 \\
& 0.734 & 0.809 & 0.677 & 0.777 & 0.659 & 0.770 & 0.612 & 0.749 \\
\hline \multirow{5}{*}{1.0} & 0.593 & 0.593 & 0.583 & 0.583 & 0.581 & 0.581 & 0.577 & 0.577 \\
& 0.535 & 0.535 & 0.513 & 0.513 & 0.508 & 0.508 & 0.500 & 0.500 \\
& 0.496 & 0.496 & 0.459 & 0.459 & 0.459 & 0.459 & 0.447 & 0.447 \\
& 0.359 & 0.521 & 0.321 & 0.499 & 0.312 & 0.495 & 0.289 & 0.481 \\
\hline \multirow{5}{*}{1.5} & 0.428 & 0.428 & 0.428 & 0.428 & 0.427 & 0.427 & 0.426 & 0.426 \\
& 0.374 & 0.374 & 0.366 & 0.366 & 0.363 & 0.363 & 0.359 & 0.359 \\
& 0.340 & 0.340 & 0.321 & 0.321 & 0.323 & 0.323 & 0.316 & 0.316 \\
& 0.135 & 0.338 & 0.119 & 0.329 & 0.116 & 0.327 & 0.107 & 0.320 \\
\hline \multirow{5}{*}{2.0} & 0.330 & 0.330 & 0.333 & 0.333 & 0.333 & 0.333 & 0.333 & 0.333 \\
& 0.284 & 0.284 & 0.281 & 0.281 & 0.280 & 0.280 & 0.277 & 0.277 \\
& 0.256 & 0.256 & 0.245 & 0.245 & 0.247 & 0.247 & 0.243 & 0.243 \\
& 0.009 & 0.231 & 0.003 & 0.228 & 0.002 & 0.227 & 0.000 & 0.222 \\
\hline
\end{tabular}

Table $4: d / D_{u}=1$. Relative bias of $\hat{C}_{p, n}^{\prime \prime}(u, v)$ when $(u, v)=(0,3),(0,4),(1,2)$ (top to bottom), $b=2,6$ (left to right), and $n=30$.

\begin{tabular}{|c|c|c|c|c|c|}
\hline$a$ & 0 & 0.5 & 1.0 & 1.5 & 2.0 \\
\hline & $-0.004-0.004$ & $0.025 \quad 0.025$ & 0.0220 .022 & 0.0130 .013 & $0.008 \quad 0.008$ \\
\hline & $\begin{array}{ll}-0.017 & -0.017\end{array}$ & 0.0290 .029 & $0.024 \quad 0.024$ & $0.014 \quad 0.014$ & $\begin{array}{lll}0.008 & 0.008\end{array}$ \\
\hline & $-0.061-0.014$ & $0.037 \quad 0.026$ & $0.043 \quad 0.025$ & $\begin{array}{lll}0.051 & 0.017\end{array}$ & undef. 0.0123 \\
\hline
\end{tabular}

Table $5: d / D_{u}=1.100 \times \operatorname{MSE}$ of $\hat{C}_{p, n}^{\prime \prime}(u, v)$ when $(u, v)=(0,3),(0,4),(1,2)$ (top to bottom), and $n=30$.

\begin{tabular}{|l|c|c|c|c|c|}
\hline \multicolumn{1}{|c|}{$a$} & 0 & 0.5 & 1.0 & 1.5 & 2.0 \\
$b$ & & & & & \\
\hline 2 & 0.857 & 0.828 & 0.258 & 0.074 & 0.026 \\
& 0.904 & 0.907 & 0.234 & 0.061 & 0.021 \\
& 1.204 & 1.212 & 0.401 & 0.121 & 0.044 \\
\hline 3 & 1.927 & 1.863 & 0.581 & 0.166 & 0.059 \\
& 2.035 & 2.040 & 0.526 & 0.136 & 0.047 \\
& 2.266 & 2.303 & 0.804 & 0.249 & 0.092 \\
\hline 4 & 3.426 & 3.312 & 1.032 & 0.295 & 0.106 \\
& 3.617 & 3.627 & 0.935 & 0.242 & 0.084 \\
& 3.748 & 3.762 & 1.349 & 0.424 & 0.158 \\
\hline 5 & 5.354 & 5.175 & 1.613 & 0.460 & 0.165 \\
& 5.652 & 5.668 & 1.461 & 0.379 & 0.131 \\
& 5.649 & 5.589 & 2.037 & 0.646 & 0.242 \\
\hline 6 & 7.709 & 7.452 & 2.323 & 0.663 & 0.238 \\
& 8.139 & 8.161 & 2.104 & 0.545 & 0.188 \\
& 7.971 & 7.783 & 2.867 & 0.915 & 0.344 \\
\hline
\end{tabular}


Table $6: d / D_{u}=4 / 3$. Relative bias of $\hat{C}_{p, n}^{\prime \prime}(u, v)$ when the process is on target and $n=30$.

\begin{tabular}{|c|c|c|c|c|c|c|}
\hline$b=2$ & \multicolumn{6}{|c|}{$v$} \\
\hline$u$ & 0 & 1 & 2 & 3 & 4 & 5 \\
\hline 0 & & 0.023 & 0.005 & -0.011 & -0.025 & -0.037 \\
\hline 1 & -0.017 & -0.035 & -0.051 & -0.065 & -0.078 & -0.089 \\
\hline 2 & -0.077 & -0.094 & -0.107 & -0.120 & -0.130 & -0.140 \\
\hline 3 & -0.138 & -0.152 & -0.163 & -0.174 & -0.183 & -0.192 \\
\hline 4 & -0.199 & -0.210 & -0.220 & -0.228 & -0.236 & -0.243 \\
\hline 5 & -0.260 & -0.268 & -0.276 & -0.283 & -0.289 & -0.295 \\
\hline
\end{tabular}

\begin{tabular}{|c|c|c|c|c|c|c|}
\hline$b=6$ & \multicolumn{7}{|c|}{$v$} \\
\hline$u$ & 0 & 1 & 2 & 3 & 4 & 5 \\
\hline 0 & & 0.023 & 0.005 & -0.011 & -0.025 & -0.037 \\
\hline 1 & 0.024 & 0.003 & -0.014 & -0.029 & -0.042 & -0.055 \\
\hline 2 & 0.004 & -0.016 & -0.033 & -0.047 & -0.060 & -0.072 \\
\hline 3 & -0.017 & -0.035 & -0.051 & -0.065 & -0.078 & -0.089 \\
\hline 4 & -0.037 & -0.055 & -0.070 & -0.083 & -0.095 & -0.106 \\
\hline 5 & -0.057 & -0.074 & -0.089 & -0.104 & -0.113 & -0.123 \\
\hline
\end{tabular}

Table $7: d / D_{u}=4 / 3.100 \times$ MSE of $\hat{C}_{p, n}^{\prime \prime}(u, v)$ when the process is on target and $n=30$.

\begin{tabular}{|c|c|c|c|c|c|c|}
\hline$b=2$ & \multicolumn{7}{|c|}{$v$} \\
\hline$u$ & 0 & 1 & 2 & 3 & 4 & 5 \\
\hline 0 & & 0.872 & 0.868 & 0.919 & 1.001 & 1.101 \\
\hline 1 & 0.928 & 1.031 & 1.176 & 1.338 & 1.505 & 1.675 \\
\hline 2 & 1.422 & 1.674 & 1.919 & 2.153 & 2.378 & 2.593 \\
\hline 3 & 2.475 & 2.801 & 3.095 & 3.366 & 3.619 & 3.856 \\
\hline 4 & 4.088 & 4.412 & 4.707 & 4.977 & 5.228 & 5.463 \\
\hline 5 & 6.261 & 6.508 & 6.752 & 6.985 & 7.205 & 7.414 \\
\hline
\end{tabular}

\begin{tabular}{|c|c|c|c|c|c|c|}
\hline$b=6$ & \multicolumn{6}{|c|}{$v$} \\
\hline$u$ & 0 & 1 & 2 & 3 & 4 & 5 \\
\hline 0 & & 7.850 & 7.815 & 8.274 & 9.007 & 9.905 \\
\hline 1 & 8.184 & 7.842 & 8.305 & 9.131 & 10.152 & 11.283 \\
\hline 2 & 7.986 & 8.319 & 9.229 & 10.386 & 11.665 & 13.005 \\
\hline 3 & 8.349 & 9.279 & 10.587 & 12.038 & 13.546 & 15.072 \\
\hline 4 & 9.270 & 10.724 & 12.379 & 14.088 & 15.796 & 17.482 \\
\hline 5 & 10.752 & 12.653 & 14.606 & 16.534 & 18.414 & 20.238 \\
\hline
\end{tabular}


Table 8: $d / D_{u}=4 / 3$. Sensitivity of $\hat{C}_{p, n}^{\prime \prime}(u, v)$ and $C_{p}^{\prime \prime}(u, v)$, when $(u, v)=(0,2),(0,3),(0,4)$, $(1,2)$ (top to bottom) and when $b=2,6$ (left to right)

\begin{tabular}{|c|c|c|c|c|c|}
\hline & \multicolumn{4}{|c|}{ Sensitivity of $\hat{C}_{p, n}^{\prime \prime}(u, v)$} & $\begin{array}{c}\text { Sensitivity of } \\
C_{p}^{\prime \prime}(u, v)\end{array}$ \\
\hline$a$ & & 10 & $\mathrm{n}=30$ & $\mathrm{n}=50$ & \\
\hline \multirow{4}{*}{-2.0} & 0.405 & 0.405 & $0.406 \quad 0.406$ & $0.405 \quad 0.405$ & $0.404 \quad 0.404$ \\
\hline & 0.352 & 0.352 & 0.3460 .346 & 0.3440 .344 & $\begin{array}{lll}0.339 & 0.339\end{array}$ \\
\hline & 0.319 & 0.319 & $0.308 \quad 0.308$ & 0.3050 .305 & 0.2980 .298 \\
\hline & 0.184 & 0.335 & $\begin{array}{lll}0.174 & 0.331\end{array}$ & 0.1690 .330 & 0.1620 .323 \\
\hline \multirow{4}{*}{-1.5} & 0.515 & 0.515 & 0.5120 .512 & $\begin{array}{lll}0.511 & 0.511\end{array}$ & $0.508 \quad 0.508$ \\
\hline & 0.456 & 0.456 & $0.444 \quad 0.444$ & $0.440 \quad 0.440$ & $0.434 \quad 0.434$ \\
\hline & 0.417 & 0.417 & $0.400 \quad 0.400$ & 0.3940 .394 & 0.3850 .385 \\
\hline & 0.320 & 0.454 & $\begin{array}{lll}0.301 & 0.444\end{array}$ & $0.295 \quad 0.441$ & $0.279 \quad 0.432$ \\
\hline \multirow{4}{*}{-1.0} & 0.685 & 0.685 & 0.6720 .672 & $\begin{array}{lll}0.668 & 0.668\end{array}$ & 0.6620 .662 \\
\hline & 0.629 & 0.629 & 0.6030 .603 & $\begin{array}{lll}0.597 & 0.597\end{array}$ & 0.5850 .585 \\
\hline & 0.590 & 0.590 & $0.555 \quad 0.555$ & 0.5460 .546 & $0.530 \quad 0.530$ \\
\hline & 0.540 & 0.640 & $\begin{array}{lll}0.502 & 0.617\end{array}$ & 0.4840 .611 & 0.4640 .596 \\
\hline \multirow{4}{*}{-0.5} & 0.909 & 0.909 & 0.8870 .887 & $\begin{array}{|ll|}0.881 & 0.881 \\
\end{array}$ & $0.870 \quad 0.870$ \\
\hline & 0.885 & 0.885 & $0.850 \quad 0.850$ & $0.840 \quad 0.840$ & 0.8220 .822 \\
\hline & 0.866 & 0.866 & 0.8190 .819 & 0.8060 .806 & $0.781 \quad 0.781$ \\
\hline & 0.857 & 0.892 & $0.802 \quad 0.859$ & 0.7850 .850 & $\begin{array}{lll}0.740 & 0.827\end{array}$ \\
\hline \multirow{4}{*}{0.5} & 0.764 & 0.764 & $0.744 \quad 0.744$ & $\begin{array}{|ll|}0.738 & 0.738 \\
\end{array}$ & $0.728 \quad 0.728$ \\
\hline & 0.724 & 0.724 & 0.6860 .686 & 0.6750 .675 & 0.6550 .655 \\
\hline & 0.696 & 0.696 & $0.643 \quad 0.643$ & 0.6280 .628 & $0.600 \quad 0.600$ \\
\hline & 0.658 & 0.731 & $0.603 \quad 0.699$ & $\begin{array}{lll}0.586 & 0.689 \\
\end{array}$ & 0.5460 .667 \\
\hline \multirow{4}{*}{1.0} & 0.491 & 0.491 & $0.477 \quad 0.477$ & $0.474 \quad 0.474$ & $0.468 \quad 0.468$ \\
\hline & 0.436 & 0.436 & $0.412 \quad 0.412$ & $\begin{array}{lll}0.407 & 0.407\end{array}$ & $0.397 \quad 0.397$ \\
\hline & 0.400 & 0.400 & $\begin{array}{lll}0.370 & 0.370\end{array}$ & $\begin{array}{ll}0.363 & 0.363\end{array}$ & 0.3510 .351 \\
\hline & 0.292 & 0.429 & 0.2590 .407 & 0.2450 .402 & $0.234 \quad 0.390$ \\
\hline \multirow{4}{*}{1.5} & 0.339 & 0.339 & 0.3360 .336 & 0.3350 .335 & 0.3330 .333 \\
\hline & 0.293 & 0.293 & $0.284 \quad 0.284$ & $\begin{array}{lll}0.282 & 0.282\end{array}$ & $0.277 \quad 0.277$ \\
\hline & 0.264 & 0.264 & $0.252 \quad 0.252$ & 0.2490 .249 & $0.243 \quad 0.243$ \\
\hline & 0.105 & 0.266 & $0.093 \quad 0.258$ & $0.090 \quad 0.256$ & $0.083 \quad 0.250$ \\
\hline \multirow{4}{*}{2.0} & 0.256 & 0.256 & $0.257 \quad 0.257$ & $\begin{array}{lll}0.257 & 0.257\end{array}$ & 0.2560 .256 \\
\hline & 0.219 & 0.219 & 0.2160 .216 & $0.214 \quad 0.214$ & $0.212 \quad 0.212$ \\
\hline & 0.197 & 0.197 & 0.1910 .191 & 0.1890 .189 & 0.1840 .184 \\
\hline & 0.007 & 0.178 & $0.002 \quad 0.175$ & 0.0010 .174 & $0.000 \quad 0.171$ \\
\hline
\end{tabular}

Table 9: $d / D_{u}=4 / 3$. Relative bias of $\hat{C}_{p, n}^{\prime \prime}(u, v)$ when $(u, v)=(0,3),(0,4),(1,2)$ (top to bottom), $b=2,6$ (left to right), and $n=30$.

\begin{tabular}{|c|c|c|c|c|c|c|c|c|c|}
\hline$b^{a}$ & -2.0 & -1.5 & -1.0 & -0.5 & 0 & 0.5 & 1.0 & 1.5 & 2.0 \\
\hline 2 & $\begin{array}{l}0.009 \\
0.008 \\
0.020\end{array}$ & $\begin{array}{l}0.013 \\
0.013 \\
0.022\end{array}$ & $\begin{array}{l}0.020 \\
0.021 \\
0.028\end{array}$ & $\begin{array}{l}0.022 \\
0.023 \\
0.029\end{array}$ & $\begin{array}{l}-0.011 \\
-0.025 \\
-0.051\end{array}$ & $\begin{array}{l}0.036 \\
0.045 \\
0.048\end{array}$ & $\begin{array}{l}0.026 \\
0.028 \\
0.051\end{array}$ & $\begin{array}{l}0.014 \\
0.014 \\
0.055\end{array}$ & $\begin{array}{l}0.008 \\
0.008 \\
\text { undef. }\end{array}$ \\
\hline 6 & $\begin{array}{l}0.009 \\
0.008 \\
0.011\end{array}$ & $\begin{array}{l}0.013 \\
0.013 \\
0.015\end{array}$ & $\begin{array}{l}0.020 \\
0.021 \\
0.021\end{array}$ & $\begin{array}{l}0.022 \\
0.023 \\
0.025\end{array}$ & $\begin{array}{l}-0.011 \\
-0.025 \\
-0.014\end{array}$ & $\begin{array}{l}0.036 \\
0.045 \\
0.033\end{array}$ & $\begin{array}{l}0.026 \\
0.028 \\
0.029 \\
\end{array}$ & $\begin{array}{l}0.014 \\
0.014 \\
0.018\end{array}$ & \begin{tabular}{|l|}
0.008 \\
0.008 \\
0.012 \\
\end{tabular} \\
\hline
\end{tabular}


Table 10:d/D $=4 / 3.100 \times \operatorname{MSE}$ of $\hat{C}_{p, n}^{\prime \prime}(u, v)$ when $(u, v)=(0,3),(0,4),(1,2)$ (top to bottom), and $n=30$.

\begin{tabular}{|l|c|c|c|c|c|c|c|c|c|}
\hline \multirow{3}{*}{$a$} & -2.0 & -1.5 & -1.0 & -0.5 & 0 & 0.5 & 1.0 & 1.5 & 2.0 \\
\hline 2 & & & & & & & & & \\
\hline & 0.037 & 0.096 & 0.287 & 0.728 & 0.919 & 0.987 & 0.206 & 0.049 & 0.016 \\
& 0.030 & 0.081 & 0.270 & 0.787 & 1.001 & 1.057 & 0.177 & 0.039 & 0.013 \\
& 0.057 & 0.141 & 0.389 & 0.903 & 1.176 & 1.327 & 0.310 & 0.077 & 0.026 \\
\hline 3 & 0.083 & 0.216 & 0.646 & 1.637 & 2.069 & 2.221 & 0.464 & 0.110 & 0.037 \\
& 0.067 & 0.183 & 0.607 & 1.771 & 2.252 & 2.379 & 0.399 & 0.088 & 0.029 \\
& 0.121 & 0.298 & 0.805 & 1.829 & 2.307 & 2.607 & 0.644 & 0.165 & 0.056 \\
\hline 4 & 0.148 & 0.384 & 1.148 & 2.910 & 3.677 & 3.949 & 0.825 & 0.196 & 0.065 \\
& 0.120 & 0.325 & 1.078 & 3.149 & 4.003 & 4.230 & 0.708 & 0.156 & 0.051 \\
& 0.210 & 0.513 & 1.374 & 3.095 & 3.872 & 4.324 & 1.101 & 0.285 & 0.098 \\
\hline 5 & 0.232 & 0.600 & 1.794 & 4.547 & 5.747 & 6.170 & 1.289 & 0.306 & 0.102 \\
& 0.187 & 0.509 & 1.685 & 4.921 & 6.255 & 6.609 & 1.107 & 0.244 & 0.079 \\
& 0.323 & 0.787 & 2.096 & 4.699 & 5.871 & 6.479 & 1.680 & 0.438 & 0.151 \\
\hline 6 & 0.334 & 0.864 & 2.584 & 6.548 & 8.274 & 8.885 & 1.856 & 0.441 & 0.147 \\
& 0.269 & 0.732 & 2.426 & 7.086 & 9.007 & 9.517 & 1.594 & 0.351 & 0.114 \\
& 0.461 & 1.120 & 2.973 & 6.643 & 8.305 & 9.071 & 2.381 & 0.624 & 0.216 \\
\hline
\end{tabular}

Table 11: $d / D_{u}=2$. Relative bias of $\hat{C}_{p, n}^{\prime \prime}(u, v)$ when the process is on target and $n=30$.

\begin{tabular}{|c|c|c|c|c|c|c|}
\hline$b=2$ & \multicolumn{7}{|c|}{$v$} \\
\hline$u$ & 0 & 1 & 2 & 3 & 4 & 5 \\
\hline 0 & & 0.009 & -0.016 & -0.036 & -0.053 & -0.068 \\
\hline 1 & -0.006 & -0.037 & -0.060 & -0.078 & -0.093 & -0.107 \\
\hline 2 & -0.057 & -0.083 & -0.103 & -0.119 & -0.133 & -0.145 \\
\hline 3 & -0.108 & -0.130 & -0.147 & -0.161 & -0.173 & -0.183 \\
\hline 4 & -0.159 & -0.176 & -0.190 & -0.202 & -0.213 & -0.222 \\
\hline 5 & -0.209 & -0.222 & -0.234 & -0.244 & -0.252 & -0.260 \\
\hline
\end{tabular}

\begin{tabular}{|c|c|c|c|c|c|c|}
\hline$b=6$ & \multicolumn{6}{|c|}{$v$} \\
\hline$u$ & 0 & 1 & 2 & 3 & 4 & 5 \\
\hline 0 & & 0.009 & -0.016 & -0.036 & -0.053 & -0.068 \\
\hline 1 & 0.028 & -0.006 & -0.030 & -0.050 & -0.067 & -0.081 \\
\hline 2 & 0.011 & -0.022 & -0.045 & -0.064 & -0.080 & -0.094 \\
\hline 3 & -0.001 & -0.037 & -0.060 & -0.078 & -0.093 & -0.107 \\
\hline 4 & -0.023 & -0.053 & -0.074 & -0.091 & -0.106 & -0.119 \\
\hline 5 & -0.040 & -0.068 & -0.089 & -0.105 & -0.120 & -0.132 \\
\hline
\end{tabular}


Table $12: d / D_{u}=2.100 \times$ MSE of $\hat{C}_{p, n}^{\prime \prime}(u, v)$ when the process is on target and $n=30$.

\begin{tabular}{|c|c|c|c|c|c|c|}
\hline$b=2$ & \multicolumn{7}{|c|}{$v$} \\
\hline$u$ & 0 & 1 & 2 & 3 & 4 & 5 \\
\hline 0 & & 0.914 & 1.063 & 1.265 & 1.482 & 1.702 \\
\hline 1 & 0.934 & 1.183 & 1.487 & 1.785 & 2.069 & 2.337 \\
\hline 2 & 1.332 & 1.791 & 2.194 & 2.552 & 2.876 & 3.172 \\
\hline 3 & 2.187 & 2.739 & 3.184 & 3.565 & 3.903 & 4.208 \\
\hline 4 & 3.499 & 4.027 & 4.456 & 4.824 & 5.150 & 5.445 \\
\hline 5 & 5.269 & 5.654 & 6.011 & 6.329 & 6.618 & 6.881 \\
\hline
\end{tabular}

\begin{tabular}{|c|c|c|c|c|c|c|}
\hline$b=6$ & \multicolumn{6}{|c|}{$v$} \\
\hline$u$ & 0 & 1 & 2 & 3 & 4 & 5 \\
\hline 0 & & 8.230 & 9.565 & 11.384 & 13.341 & 15.316 \\
\hline 1 & 8.305 & 8.697 & 10.555 & 12.699 & 14.880 & 17.021 \\
\hline 2 & 8.127 & 9.503 & 11.828 & 14.260 & 16.639 & 18.926 \\
\hline 3 & 8.406 & 10.648 & 13.383 & 16.067 & 18.619 & 21.031 \\
\hline 4 & 9.142 & 12.134 & 15.221 & 18.121 & 20.819 & 23.337 \\
\hline 5 & 10.336 & 13.959 & 17.342 & 20.420 & 23.239 & 25.844 \\
\hline
\end{tabular}


Table $13: d / D_{u}=2$. Sensitivity of $\hat{C}_{p, n}^{\prime \prime}(u, v)$ and $C_{p}^{\prime \prime}(u, v)$, when $(u, v)=(0,1),(0,2),(1,1)$, $(1,2)$ (top to bottom), and $b=2,6$ (left to right)

\begin{tabular}{|c|c|c|c|c|}
\hline & \multicolumn{3}{|c|}{ Sensitivity of $\hat{C}_{p, n}^{\prime \prime}(u, v)$} & \multirow{2}{*}{$\begin{array}{c}\text { Sensitivity of } \\
C_{p}^{\prime \prime}(u, v)\end{array}$} \\
\hline$a$ & $\mathrm{n}=10$ & $\mathrm{n}=30$ & $\mathrm{n}=50$ & \\
\hline \multirow{4}{*}{-2.0} & 0.5960 .596 & 0.6020 .602 & $0.602 \quad 0.602$ & $0.600 \quad 0.600$ \\
\hline & $\begin{array}{lll}0.487 & 0.487\end{array}$ & 0.4810 .481 & $0.477 \quad 0.477$ & $0.468 \quad 0.468$ \\
\hline & $0.432 \quad 0.544$ & $0.422 \quad 0.543$ & $0.417 \quad 0.541$ & $0.400 \quad 0.533$ \\
\hline & $0.352 \quad 0.444$ & 0.3360 .434 & $0.330 \quad 0.429$ & 0.3120 .416 \\
\hline \multirow{4}{*}{-1.5} & $0.713 \quad 0.713$ & $0.712 \quad 0.712$ & $0.711 \quad 0.711$ & 0.6770 .677 \\
\hline & 0.6090 .609 & 0.5950 .595 & 0.5890 .589 & $\begin{array}{lll}0.577 & 0.577\end{array}$ \\
\hline & $\begin{array}{lll}0.581 & 0.671\end{array}$ & 0.5620 .664 & 0.5540 .660 & 0.5300 .648 \\
\hline & $0.495 \quad 0.573$ & $0.468 \quad 0.554$ & $0.459 \quad 0.547$ & $0.433 \quad 0.529$ \\
\hline \multirow{4}{*}{-1.0} & $0.858 \quad 0.858$ & $0.844 \quad 0.844$ & $0.840 \quad 0.840$ & 0.8320 .832 \\
\hline & $0.782 \quad 0.782$ & $0.754 \quad 0.754$ & $0.745 \quad 0.745$ & $\begin{array}{ll}0.728 & 0.728\end{array}$ \\
\hline & $0.776 \quad 0.832$ & 0.7390 .810 & 0.7270 .803 & 0.6930 .786 \\
\hline & $0.707 \quad 0.758$ & $\begin{array}{lll}0.659 & 0.723 \\
\end{array}$ & $0.645 \quad 0.713$ & 0.6060 .687 \\
\hline \multirow{4}{*}{-0.5} & $\begin{array}{ll}0.999 & 0.999\end{array}$ & $\begin{array}{ll}0.970 & 0.970\end{array}$ & $0.962 \quad 0.962$ & 0.9490 .949 \\
\hline & 0.9850 .985 & 0.9430 .943 & $0.930 \quad 0.930$ & 0.9050 .905 \\
\hline & $0.986 \quad 0.994$ & 0.9330 .958 & 0.9160 .947 & $0.870 \quad 0.922$ \\
\hline & $0.970 \quad 0.980$ & $0.906 \quad 0.931$ & 0.8850 .915 & 0.8290 .875 \\
\hline \multirow{4}{*}{0.5} & 0.7340 .734 & $\begin{array}{ll}0.721 & 0.721\end{array}$ & $0.717 \quad 0.717$ & $0.707 \quad 0.707$ \\
\hline & 0.6580 .658 & 0.6160 .616 & 0.6030 .603 & $\begin{array}{lll}0.577 & 0.577\end{array}$ \\
\hline & 0.6230 .699 & 0.5790 .675 & 0.5650 .667 & $0.530 \quad 0.648$ \\
\hline & 0.5650 .629 & $0.497 \quad 0.578$ & $0.477 \quad 0.562$ & $0.433 \quad 0.529$ \\
\hline \multirow{4}{*}{1.0} & $0.462 \quad 0.462$ & $0.454 \quad 0.454$ & $0.452 \quad 0.452$ & $0.447 \quad 0.447$ \\
\hline & $0.370 \quad 0.370$ & $0.348 \quad 0.348$ & 0.3430 .343 & 0.3330 .333 \\
\hline & $\begin{array}{lll}0.271 & 0.401\end{array}$ & 0.2440 .386 & 0.2380 .382 & 0.2240 .373 \\
\hline & $0.219 \quad 0.322$ & $\begin{array}{lll}0.188 & 0.297\end{array}$ & $\begin{array}{ll}0.181 & 0.290\end{array}$ & $0.167 \quad 0.278$ \\
\hline \multirow{4}{*}{1.5} & 0.3160 .316 & $\begin{array}{ll}0.318 & 0.318\end{array}$ & $0.317 \quad 0.317$ & 0.3160 .316 \\
\hline & $0.243 \quad 0.243$ & $\begin{array}{lll}0.237 & 0.237\end{array}$ & $0.234 \quad 0.234$ & 0.2290 .229 \\
\hline & $0.096 \quad 0.247$ & $0.087 \quad 0.243$ & $0.084 \quad 0.242$ & 0.0790 .236 \\
\hline & $0.074 \quad 0.189$ & $\begin{array}{lll}0.065 & 0.181 \\
\end{array}$ & $0.062 \quad 0.178$ & $0.057 \quad 0.172$ \\
\hline \multirow{4}{*}{2.0} & $0.238 \quad 0.238$ & $0.242 \quad 0.242$ & $0.243 \quad 0.243$ & $0.243 \quad 0.243$ \\
\hline & 0.1810 .181 & $0.178 \quad 0.178$ & 0.1770 .177 & $0.174 \quad 0.174$ \\
\hline & 0.0060 .165 & 0.0020 .165 & $0.000 \quad 0.164$ & $0.000 \quad 0.162$ \\
\hline & $0.005 \quad 0.125$ & $\begin{array}{lll}0.002 & 0.121\end{array}$ & $0.001 \quad 0.120$ & 0.0000 .116 \\
\hline
\end{tabular}


Tableau $14: d / D_{u}=2$. Relative bias of $\hat{C}_{p, n}^{\prime \prime}(u, v)$ when $(u, v)=(0,2),(1,1),(1,2)$ (top to bottom), and $n=30$.

\begin{tabular}{|c|c|c|c|c|c|c|c|c|c|}
\hline$a$ & -2.0 & -1.5 & -1.0 & -0.5 & 0 & 0.5 & 1.0 & 1.5 & 2.0 \\
\hline \multirow{3}{*}{2} & 0.009 & 01 & 020 & 0.026 & -0.016 & 0.050 & 0.029 & 0.014 & 0.008 \\
\hline & 0.015 & 0.019 & 0.026 & 0.033 & -0.037 & 0.051 & 0.052 & 0.055 & undef. \\
\hline & 0.013 & 0.017 & 0.023 & 0.028 & -0.059 & 0.080 & 0.061 & 0.058 & undef. \\
\hline \multirow{3}{*}{6} & 0.009 & 0.014 & 0.020 & 0.026 & -0.016 & 0.050 & 0.029 & 0.014 & 0.008 \\
\hline & 0.013 & 0.018 & 0.025 & 0.032 & -0.006 & 0.035 & 0.030 & 0.018 & 0.012 \\
\hline & 0.010 & 0.015 & 0.021 & 0.026 & -0.030 & 0.058 & 0.035 & 0.019 & 0.013 \\
\hline
\end{tabular}

Tableau $15: d / D_{u}=2.100 \times \operatorname{MSE}$ of $\hat{C}_{p, n}^{\prime \prime}(u, v)$ when $(u, v)=(0,2),(1,1),(1,2)$ (top to bottom), and $n=30$.

\begin{tabular}{|l|c|c|c|c|c|c|c|c|c|}
\hline \multirow{3}{*}{$a$} & -2.0 & -1.5 & -1.0 & -0.5 & 0 & 0.5 & 1.0 & 1.5 & 2.0 \\
\hline 2 & & & & & & & & & \\
\hline & 0.062 & 0.139 & 0.327 & 0.670 & 1.063 & 1.082 & 0.165 & 0.035 & 0.011 \\
& 0.100 & 0.202 & 0.413 & 0.749 & 1.183 & 1.349 & 0.291 & 0.070 & 0.023 \\
& 0.067 & 0.153 & 0.368 & 0.752 & 1.487 & 1.416 & 0.187 & 0.038 & 0.012 \\
\hline 3 & 0.139 & 0.313 & 0.737 & 1.507 & 2.391 & 2.435 & 0.372 & 0.080 & 0.026 \\
& 0.220 & 0.445 & 0.912 & 1.651 & 2.376 & 2.669 & 0.609 & 0.150 & 0.051 \\
& 0.147 & 0.332 & 0.792 & 1.617 & 2.957 & 2.920 & 0.404 & 0.084 & 0.027 \\
\hline 4 & 0.247 & 0.556 & 1.309 & 2.679 & 4.251 & 4.329 & 0.661 & 0.141 & 0.046 \\
& 0.389 & 0.787 & 1.611 & 2.916 & 4.025 & 4.444 & 1.044 & 0.261 & 0.088 \\
& 0.257 & 0.581 & 1.380 & 2.818 & 4.958 & 4.965 & 0.704 & 0.147 & 0.047 \\
\hline 5 & 0.387 & 0.868 & 2.046 & 4.186 & 6.642 & 6.764 & 1.033 & 0.221 & 0.071 \\
& 0.606 & 1.226 & 2.511 & 4.545 & 6.132 & 6.672 & 1.596 & 0.401 & 0.136 \\
& 0.398 & 0.899 & 2.132 & 4.353 & 7.491 & 7.552 & 1.086 & 0.228 & 0.073 \\
\hline 6 & 0.557 & 1.250 & 2.946 & 6.028 & 9.565 & 9.740 & 1.487 & 0.318 & 0.103 \\
& 0.872 & 1.765 & 3.613 & 6.536 & 8.696 & 9.355 & 2.264 & 0.572 & 0.195 \\
& 0.570 & 1.287 & 3.048 & 6.223 & 10.555 & 10.679 & 1.551 & 0.327 & 0.104 \\
\hline
\end{tabular}


Table 16: $d / D_{u}=4$. Relative bias of $\hat{C}_{p, n}^{\prime \prime}(u, v)$ when the process is on target and $n=30$.

\begin{tabular}{|c|c|c|c|c|c|c|}
\hline$b=2$ & \multicolumn{7}{|c|}{$v$} \\
\hline$u$ & 0 & 1 & 2 & 3 & 4 & 5 \\
\hline 0 & & -0.041 & -0.082 & -0.111 & -0.133 & -0.151 \\
\hline 1 & 0.001 & -0.074 & -0.112 & -0.138 & -0.158 & -0.174 \\
\hline 2 & -0.043 & -0.107 & -0.141 & -0.164 & -0.183 & -0.198 \\
\hline 3 & -0.086 & -0.141 & -0.170 & -0.191 & -0.208 & -0.221 \\
\hline 4 & -0.130 & -0.174 & -0.199 & -0.218 & -0.233 & -0.245 \\
\hline 5 & -0.173 & -0.207 & -0.228 & -0.245 & -0.258 & -0.269 \\
\hline
\end{tabular}

\begin{tabular}{|c|c|c|c|c|c|c|}
\hline$b=6$ & \multicolumn{6}{|c|}{$v$} \\
\hline$u$ & 0 & 1 & 2 & 3 & 4 & 5 \\
\hline 0 & & -0.041 & -0.082 & -0.111 & -0.133 & -0.151 \\
\hline 1 & 0.030 & -0.052 & -0.092 & -0.120 & -0.141 & -0.159 \\
\hline 2 & 0.016 & -0.063 & -0.102 & -0.129 & -0.149 & -0.166 \\
\hline 3 & 0.001 & -0.074 & -0.112 & -0.138 & -0.158 & -0.174 \\
\hline 4 & -0.014 & -0.085 & -0.121 & -0.147 & -0.166 & -0.182 \\
\hline 5 & -0.028 & -0.096 & -0.131 & -0.155 & -0.174 & -0.190 \\
\hline
\end{tabular}

Table $17: d / D_{u}=4.100 \times$ MSE of $\hat{C}_{p, n}^{\prime \prime}(u, v)$ when the process is on target and $n=30$.

\begin{tabular}{|c|c|c|c|c|c|c|}
\hline$b=2$ & \multicolumn{6}{|c|}{$v$} \\
\hline$u$ & 0 & 1 & 2 & 3 & 4 & 5 \\
\hline 0 & & 1.532 & 2.344 & 3.027 & 3.604 & 4.104 \\
\hline 1 & 0.957 & 2.081 & 2.997 & 3.716 & 4.307 & 4.810 \\
\hline 2 & 1.340 & 2.821 & 3.785 & 4.512 & 5.099 & 5.595 \\
\hline 3 & 2.142 & 3.750 & 4.707 & 5.414 & 5.982 & 6.458 \\
\hline 4 & 3.365 & 4.870 & 5.764 & 6.424 & 6.954 & 7.398 \\
\hline 5 & 5.008 & 6.179 & 6.956 & 7.541 & 8.016 & 8.417 \\
\hline
\end{tabular}

\begin{tabular}{|c|c|c|c|c|c|c|}
\hline $\mathrm{b}=6$ & \multicolumn{6}{|c|}{$\mathrm{v}$} \\
\hline $\mathrm{u}$ & 0 & 1 & 2 & 3 & 4 & 5 \\
\hline 0 & & 13.783 & 21.099 & 27.241 & 32.440 & 36.933 \\
\hline 1 & 8.410 & 15.242 & 22.922 & 29.201 & 34.458 & 38.975 \\
\hline 2 & 8.300 & 16.891 & 24.881 & 31.267 & 36.565 & 41.095 \\
\hline 3 & 8.608 & 18.731 & 26.974 & 33.441 & 38.763 & 43.293 \\
\hline 4 & 9.338 & 20.760 & 29.202 & 35.721 & 41.050 & 45.569 \\
\hline 5 & 10.487 & 22.978 & 31.565 & 38.109 & 43.427 & 47.923 \\
\hline
\end{tabular}


Table $18: d / D_{u}=4$. Sensitivity of $\hat{C}_{p, n}^{\prime \prime}(u, v)$ and $C_{p}^{\prime \prime}(u, v)$, when $(u, v)=(0,1),(1,1),(2,0)$, $(3,0)$ (top to bottom), and $b=2,6$ (left to right)

\begin{tabular}{|c|c|c|c|c|}
\hline & \multicolumn{3}{|c|}{ Sensitivity of $\hat{C}_{p, n}^{\prime \prime}(u, v)$} & $\begin{array}{c}\text { Sensitivity of } \\
C_{p}^{\prime \prime}(u, v)\end{array}$ \\
\hline$a$ & $\mathrm{n}=10$ & $\mathrm{n}=30$ & $\mathrm{n}=50$ & \\
\hline \multirow{4}{*}{-2.0} & 7160.716 & 6960.696 & 0.6860 .686 & 0.6860 .686 \\
\hline & $48 \quad 0.694$ & 6190.671 & 0.6050 .659 & 0.5630 .627 \\
\hline & $\begin{array}{ll}0.835 & 0.951\end{array}$ & $\begin{array}{ll}779 & 0.931\end{array}$ & $0.763 \quad 0.925$ & 0.7140 .905 \\
\hline & $\begin{array}{lll}0.729 & 0.924\end{array}$ & 0.6530 .894 & 0.6330 .886 & 0.5720 .857 \\
\hline \multirow{4}{*}{-1.5} & 0.8390 .839 & $807 \quad 0.807$ & $\begin{array}{ll}0.793 & 0.793\end{array}$ & 0.7590 .759 \\
\hline & 910.824 & $\begin{array}{ll}747 & 0.787\end{array}$ & $0.728 \quad 0.772$ & 0.6780 .732 \\
\hline & $\begin{array}{ll}0.917 & 0.976\end{array}$ & 0.8570 .955 & $\begin{array}{lll}0.840 & 0.949\end{array}$ & 0.7860 .929 \\
\hline & $0.866 \quad 0.834$ & 0.7760 .932 & $\begin{array}{ll}0.751 & 0.923 \\
\end{array}$ & 0.6790 .893 \\
\hline \multirow{4}{*}{-1.0} & 820.982 & 0.9290 .929 & 0.9110 .911 & 0.8680 .868 \\
\hline & 0.9630 .976 & 0.8940 .918 & 0.8710 .898 & $0.806 \quad 0.848$ \\
\hline & $1.001 \quad 1.001$ & 9340.980 & 0.9160 .973 & 0.8570 .952 \\
\hline & 1.0021 .001 & 8980.969 & $0.870 \quad 0.960$ & 0.7860 .929 \\
\hline \multirow{4}{*}{-0.5} & $04 \quad 1.104$ & 1.0371 .037 & 1.0131 .013 & $0.962 \quad 0.962$ \\
\hline & 191.109 & 1.0361 .036 & 1.0061 .011 & 0.9270 .950 \\
\hline & 1.0751 .022 & .0131 .004 & 0.9930 .998 & $0.928 \quad 0.976$ \\
\hline & $22 \quad 1.034$ & 1.0201 .006 & 0.9890 .996 & 0.8930 .964 \\
\hline \multirow{4}{*}{-0.1} & 1.0561 .056 & .0451 .045 & 1.0371 .037 & $\begin{array}{ll}0.998 & 0.998\end{array}$ \\
\hline & 71.059 & $057 \quad 1.049$ & 1.0481 .040 & 0.9910 .996 \\
\hline & 1.0531 .013 & 1.0331 .011 & 1.0301 .009 & 0.9860 .995 \\
\hline & $1.068 \quad 1.019$ & 1.0531 .016 & 1.0451 .014 & 0.9790 .993 \\
\hline \multirow{4}{*}{0.1} & 70.927 & 0.9230 .923 & 0.9230 .923 & 0.9280 .928 \\
\hline & 120.920 & $\begin{array}{lll}0.903 & 0.917\end{array}$ & 0.9000 .916 & 0.8820 .913 \\
\hline & $\begin{array}{lll}0.942 & 0.982\end{array}$ & $\begin{array}{lll}0.940 & 0.981\end{array}$ & 0.9380 .980 & $0.900 \quad 0.967$ \\
\hline & 0.9040 .973 & $\begin{array}{lll}0.906 & 0.971\end{array}$ & $0.903 \quad 0.970$ & $0.850 \quad 0.950$ \\
\hline \multirow{4}{*}{0.5} & 0.5640 .564 & $0.504 \quad 0.504$ & 0.4850 .485 & $0.447 \quad 0.447$ \\
\hline & 0.4840 .538 & $0.406 \quad 0.472$ & $\begin{array}{lll}0.382 & 0.451\end{array}$ & 0.3350 .410 \\
\hline & 0.5730 .872 & 0.5450 .857 & $0.535 \quad 0.852$ & $0.500 \quad 0.833$ \\
\hline & $02 \quad 0.804$ & $\begin{array}{lll}0.285 & 0.783 \\
\end{array}$ & $0.277 \quad 0.775$ & $0.250 \quad 0.750$ \\
\hline \multirow{4}{*}{1.0} & 820.282 & 0.2610 .261 & 0.2550 .255 & $0.243 \quad 0.243$ \\
\hline & $\begin{array}{lll}0.166 & 0.245\end{array}$ & $0.140 \quad 0.222$ & 0.1340 .216 & $0.121 \quad 0.202$ \\
\hline & -0.0010 .700 & $0.000 \quad 0.686$ & $0.000 \quad 0.681$ & $0.000 \quad 0.667$ \\
\hline & 0.539 & 0.522 & 0.517 & 0.500 \\
\hline \multirow{4}{*}{1.5} & $0.180 \quad 0.180$ & $0.174 \quad 0.174$ & $\begin{array}{ll}0.171 & 0.171\end{array}$ & 0.1640 .164 \\
\hline & $\begin{array}{lll}0.054 & 0.139\end{array}$ & 0.0470 .133 & 0.0450 .130 & $0.041 \quad 0.123$ \\
\hline & 0.525 & 0.514 & 0.511 & 0.500 \\
\hline & 0.269 & 0.261 & 0.258 & 0.250 \\
\hline \multirow{4}{*}{2.0} & 0.1330 .133 & 0.1300 .130 & 0.1290 .129 & $0.124 \quad 0.124$ \\
\hline & $\begin{array}{lll}0.004 & 0.091\end{array}$ & 0.0010 .088 & $\begin{array}{lll}0.001 & 0.087\end{array}$ & $0.000 \quad 0.083$ \\
\hline & 0.350 & 0.343 & 0.341 & 0.333 \\
\hline & 0.000 & 0.000 & 0.000 & 0.000 \\
\hline
\end{tabular}


Table 19: $d / D_{u}=4$. Relative bias of $\hat{C}_{p, n}^{\prime \prime}(u, v)$ when $(u, v)=(0,1),(1,1),(2,0),(3,0)$ (top to bottom), and $n=30$.

\begin{tabular}{|c|c|c|c|c|c|c|c|c|c|}
\hline$a$ & -2.0 & -1.5 & -1.0 & -0.5 & 0 & 0.5 & 1.0 & 1.5 & 2.0 \\
\hline \multirow{4}{*}{2} & 0.014 & 019 & 027 & 034 & .041 & 0.081 & 0.032 & 0.015 & .008 \\
\hline & 0.015 & 0.020 & 0.027 & 0.035 & -0.074 & 0.121 & 0.067 & 0.060 & idef. \\
\hline & 0.044 & 0.044 & 0.044 & 0.044 & -0.043 & 0.044 & undef. & & \\
\hline & 0.044 & 0.044 & 0.044 & 0.044 & -0.086 & 0.043 & & & \\
\hline \multirow{4}{*}{6} & 0.014 & 0.019 & 0.027 & 0.034 & -0.041 & 0.081 & 0.032 & 0.015 & 0.008 \\
\hline & 0.014 & 0.019 & 0.027 & 0.034 & -0.052 & 0.092 & 0.039 & 0.020 & 0.013 \\
\hline & 0.044 & 0.044 & 0.044 & 0.044 & 0.016 & 0.044 & 0.044 & 0.044 & 0.044 \\
\hline & 0.044 & 0.044 & 0.044 & 0.044 & 0.001 & 0.044 & 0.044 & 0.044 & undef. \\
\hline
\end{tabular}

Table $20: d / D_{u}=4.100 \times \operatorname{MSE}$ of $\hat{C}_{p, n}^{\prime \prime}(u, v)$ when $(u, v)=(0,1),(1,1),(2,0),(3,0)$ (top to bottom), and $n=30$.

\begin{tabular}{|l|c|c|c|c|c|c|c|c|c|}
\hline \multirow{3}{*}{$a$} & -2.0 & -1.5 & -1.0 & -0.5 & 0 & 0.5 & 1.0 & 1.5 & 2.0 \\
\hline 2 & & & & & & & & & \\
& 0.123 & 0.238 & 0.456 & 0.765 & 1.531 & 1.143 & 0.102 & 0.019 & 0.006 \\
& 0.118 & 0.230 & 0.444 & 0.755 & 2.081 & 1.357 & 0.109 & 0.020 & 0.006 \\
& 0.540 & 0.647 & 0.763 & 0.889 & 1.340 & 1.883 & 1.646 & & \\
\hline 3 & 0.400 & 0.533 & 0.689 & 0.865 & 2.142 & 3.742 & & & \\
\hline & 0.276 & 0.536 & 1.027 & 1.721 & 3.446 & 2.571 & 0.230 & 0.043 & 0.013 \\
& 0.268 & 0.521 & 1.005 & 1.702 & 4.223 & 2.887 & 0.241 & 0.045 & 0.014 \\
& 1.498 & 1.676 & 1.863 & 2.059 & 2.334 & 2.628 & 1.894 & 1.646 & \\
& 1.216 & 1.456 & 1.718 & 1.999 & 3.014 & 4.238 & 3.704 & & \\
\hline 4 & 0.491 & 0.952 & 1.825 & 3.060 & 6.126 & 4.571 & 0.409 & 0.077 & 0.024 \\
& 0.480 & 0.932 & 1.795 & 3.032 & 7.130 & 4.988 & 0.423 & 0.079 & 0.024 \\
& 2.953 & 3.201 & 3.460 & 3.726 & 3.826 & 3.869 & 2.640 & 1.894 & 1.646 \\
& 2.529 & 2.875 & 3.243 & 3.630 & 4.382 & 5.230 & 3.952 & & \\
\hline 5 & 0.767 & 1.488 & 2.852 & 4.781 & 9.572 & 7.142 & 0.639 & 0.120 & 0.037 \\
& 0.753 & 1.462 & 2.813 & 4.745 & 10.803 & 7.660 & 0.657 & 0.122 & 0.038 \\
& 4.904 & 5.224 & 5.553 & 5.889 & 5.814 & 5.607 & 3.881 & 2.640 & 1.894 \\
\hline 6 & 4.338 & 4.790 & 5.266 & 5.758 & 6.247 & 6.719 & 4.697 & 3.766 & \\
\hline & 1.104 & 2.142 & 4.106 & 6.884 & 13.783 & 10.285 & 0.921 & 0.173 & 0.054 \\
& 1.087 & 2.111 & 4.059 & 6.840 & 15.242 & 10.904 & 0.942 & 0.176 & 0.054 \\
& 7.352 & 7.743 & 8.143 & 8.550 & 8.300 & 7.842 & 5.620 & 3.881 & 2.640 \\
& 6.644 & 7.203 & 7.785 & 8.383 & 8.608 & 8.705 & 5.939 & 4.262 & 3.704 \\
\hline
\end{tabular}




\section{References}

Abramowitz, M., and Stegun, I. A. (1965). Handbook of Mathematical functions with formulas, graphs, and mathematical tables. Dover publications, New York.

Chen, K. S. and Pearn, W. L. (2001). Capability indices for processes with asymmetric tolerances. Journal of the Chinese Institute of Engineers, 24(5), 559-568.

Chen, K. S., Pearn, W. L. and Lin, P. C. (1999). A new generalization of $C_{p m}$ for processes with asymmetric tolerances. International Journal of Reliability, Quality and Safety Engineering, 6(4), 383-398.

Pearn, W. L. and Chen, K. S. (1998). New generalization of process capability index $C_{p k}$. Journal of Applied Statistics, 25(6), 801-810.

Pearn, W. L., Lin, P. C. and Chen, K. S. (1999). On the generalizations of the capability index $C_{p m k}$ for asymmetric tolerances", Far East Journal of Theoretical Statistics, 3, 49-66.

Pearn, W. L., Lin, P.C. and Chen, K. S. (2001). Estimating process capability index $C_{p m k}^{\prime \prime}$ for asymmetric tolerances: distributional properties, Metrika, 54, 261-279.

Pearn, W. L., Lin, P.C. and Chen, K. S. (2004). The $C_{p k}^{\prime \prime}$ index for asymetric tolerances : implications and inference, Metrika, 60, 119-136.

Vännman, K. (1995). A unified approach to capability indices, Statistica Sinica, 5, 805-820.

Vännman, K. (1997). A general class of capability indices in the case of asymmetric tolerances. Communications in Statistics - Theory and Methods, 26(10), 2381-2396. 